\title{
Author Index for Volume 65
}

\begin{tabular}{|c|c|}
\hline Abdelfattah, MS & 245,277 \\
\hline Abdel-Mageed, WM & 433 \\
\hline Adcock, AF & 3,559 \\
\hline Agrawal, R & 559 \\
\hline Ahmed, TA & 615 \\
\hline Ahn, JS & $161,215,437$ \\
\hline Ahn, Y & 179 \\
\hline Akagawa, KS & 48 \\
\hline An, L & 38 \\
\hline Ando, K & 10 \\
\hline Andolfi, A & 20 \\
\hline Anke, $\mathrm{H}$ & \\
\hline Ara, I & 19 \\
\hline Arshad, A & 28 \\
\hline Asami, Y & 21 \\
\hline Aubouy, A & 49 \\
\hline Awakawa, T & 58 \\
\hline Ayers, S & 3,559 \\
\hline Bae, K & 437 \\
\hline Bai, H & 129,433 \\
\hline Bai, N & 175 \\
\hline Balcázar, JL & 289,301 \\
\hline Becker, J & 9 \\
\hline Bednarek, E & 219 \\
\hline Bekker, OB & 223,40 \\
\hline Bera, $S$ & 49 \\
\hline Bérdy, J & 385,441 \\
\hline Bhatia, MS & 27 \\
\hline Bhatia, NM & 27 \\
\hline Blomgren, A & 397 \\
\hline Boonruangprapa, $\mathrm{T}$ & 571 \\
\hline Bozkurt-Güzel, Ç & . \\
\hline Braña, AF & 34 \\
\hline Buss, AD & 27 \\
\hline Butler, MS & 27 \\
\hline Bykov, EE & 40 \\
\hline Cai, Y & 17 \\
\hline Cao, G-Y & \\
\hline Carbajo, RJ & 34 \\
\hline Cardona, ST & \\
\hline Carr, GJ & 62 \\
\hline Cerniglia, CE & 17 \\
\hline Chai, D & 17 \\
\hline Chaudhary, M & 63 \\
\hline Chen, A-J & 14 \\
\hline Chen, $\mathrm{C}$ & 43 \\
\hline Chen, $\mathrm{H}$ & 143 \\
\hline Chen, J & 517 \\
\hline Chen, L & 14 \\
\hline Chen, R-X & 327 \\
\hline Chen, S-C & 31 \\
\hline Chen, X-D & 14 \\
\hline Chen, X & $229,271,51$ \\
\hline
\end{tabular}

Chen, Y 479

Chen, Z 517

Cheng, J 307, 531

Cho, GY

Choi, SU

Chu, $\mathrm{H}$

Cimmino, A

Clarke, $\mathrm{M}$

Colombo, D

Cong, C

Constant, $\mathrm{P}$

Cordero-Ampuero, J

Cui, W

Daffé, M

Dahlgren, MK

Dai, $\mathrm{H}$

Danilenko, VN

Daram, D

Darveaux, BA

de Blanco, EJC

De Mieri, $M$

del Prado, G

Demain, AL

Dezhenkova, LG

Dhondikubeer, $\mathrm{R}$

Ding, F

Ding, $G$

Donadio, $S$

Drescher, KM

Du Plessis, LH

Duangmal, K

Duran, N

Eakley, NM

Elizarov, SM

Elofsson, M

Enkh-Amgalan, J

Erkel, G

Esteban, J

Evidente, A

Fadl, AA

Falagas, ME

Fallarero, A

Fan, K

Fenical, W

Ferraboschi, $\mathrm{P}$

Fiedler, H-P

Figueroa, $\mathrm{M}$

Florez, FK

Francis, L

$\mathrm{Fu}, \mathrm{P}$

$\mathrm{Fu}, \mathrm{Y}$

Fujii, T
Fujiwara, T

Fukuda, T

Fukuyama, T

Furiga, A

Furihata, K

Gan, M

Gao, N

Geisen, U

Geng, M

Gerçeker, AA

Gómez, C

Gómez-Barrena, E

Graf, TN

Grisenti, P

Groot, $\mathrm{H}$

$\mathrm{Gu}, \mathrm{J}$

Guan, Y

Guo, D

Guo, H

Guo, L

Guo, X

Gylfe, ^

Hamada, M

Hamamoto, $\mathrm{H}$

Han, $\mathrm{H}$

Hanaki, $\mathrm{H}$

Handasah, S

Hao, X

Hara, C

Harer, SL

Hasegawa, K

Hashida, J

Hashimoto, M

Haste, NM

Hasumi, K

Havlicek, V

Hayakawa, M

$\mathrm{He}, \mathrm{F}$

$\mathrm{He}, \mathrm{N}$

$\mathrm{He}, \mathrm{Q}-\mathrm{Y}$

$\mathrm{He}, \mathrm{W}$

Heili, S

Hénocq, CP

Hensler, ME

Hidaki, M

Hino, $M$

Hirose, $\mathrm{T}$

Hopp, DC

Horinouchi, S

Hosaka, T

Hotta, K

Hou, J

Hou, Z
323

207, 565

53

499

355

513, 539

327

369

449

83

341

505

3, 559

349

461

479

513, 539

175

433

271

229

397

249, 427

53

449

527

169

513, 539

599

279

91, 361, 483

197

227

593

91, 361, 483

219

249, 373, 427

109

327

327

79

151

499

593

331

227

487

623

551

41, 323, 373

377

523

129 


\begin{tabular}{|c|c|c|c|c|c|}
\hline $\mathrm{Hu}, \mathrm{J}$ & 317 & Ki, D-W & 95 & $\mathrm{Lu}, \mathrm{Q}-\mathrm{N}$ & 509 \\
\hline Huang, H & 49 & Kim, C & 87 & Luo, $\mathrm{X}$ & 129 \\
\hline Huang, Y & 479 & Kim, MR & 215 & Lysenkova, LN & 223,405 \\
\hline Hung, H-C & 311 & Kim, M-Y & 161 & & \\
\hline Hwang, J & 237 & Kim, S-E & 95 & Ma, B-J & 165 \\
\hline Ichinoseki, S & 377 & Kirst, HA & 173 & $\mathrm{Ma}, \mathrm{S}$ & 115,383 \\
\hline Igarashi, Y & $157,207,263,355,627$ & Kitani, S & 157,627 & Ma, X & 129 \\
\hline Iida, $\mathrm{T}$ & 207,355 & Kitano, Y & 483 & $\mathrm{Ma}, \mathrm{Y}$ & 59 \\
\hline Ikeda, M & 157 & Kobayashi, Y & 53 & $\mathrm{Ma}, \mathrm{Z}$ & 317 \\
\hline Imai, Y & 323 & Kodani, S & 331 & Madhyastha, S & 73 \\
\hline Inoue, $\mathrm{H}$ & 565 & Kotzé, AF & 609 & Marwoto, B & 169 \\
\hline Inouye, $\mathrm{M}$ & 237 & Koyama, R & 335 & Masuma, R & $5,139,255,527,565$ \\
\hline Iorio, $\mathrm{M}$ & 267 & Kozerski, L & 219 & Matsuda, D & $15,255,565$ \\
\hline Irigoyen, $\mathrm{M}$ & 623 & Kroll, DJ & 3,559 & Matsui, $\mathrm{H}$ & 527 \\
\hline Isaka, $\mathrm{M}$ & 571 & Kunsági-Máté, S & 193 & Matsumoto, A & $169,197,335,491$ \\
\hline Isea-Peña, M-C & 151 & Kuzdraliñski, A & 583 & Matthew, S & 3 \\
\hline Ishibashi, M & 245,277 & & & Mazzei, E & 267 \\
\hline Ishii, $\mathrm{T}$ & 139,527 & Lan, J & 143 & McDonald, T & 153 \\
\hline Ishikawa, $\mathrm{N}$ & 377 & Lee, I-K & 87,95 & Mei, Q & 307 \\
\hline Ishiyama, A & $113,169,197$ & Lee, I-S & 437 & Méndez, C & 341 \\
\hline Ito, $\mathrm{A}$ & 263 & Lee, KR & 135,575 & Meng, J & 129 \\
\hline Jensen, PR & 593 & Li, W-J & 469 & Mongkol, $\mathrm{P}$ & 157 \\
\hline Jeong, S-J & 215 & Li, X & 383 & Mori, $\mathrm{H}$ & 227 \\
\hline Jia, C & 79 & $\mathrm{Li}, \mathrm{Y}$ & 25,523 & Mori, M & 197 \\
\hline Jia, M & 129 & $\mathrm{Li}, \mathrm{Y}-\mathrm{H}$ & 509 & Moscoso, J & 461 \\
\hline Jiang, J-D & 509 & Liang, B & 175 & Motohashi, K & 211 \\
\hline Jiao, B & 115 & Liang, D & 117 & Munoz-Acuna, U & 559 \\
\hline Jing, C & 117 & Liang, $\mathrm{H}$ & 59 & Muñoz-Camargo, C & 461 \\
\hline Jinno, $M$ & 413 & Liermann, JC & 99,473 & Murao, A & 331 \\
\hline \multirow[t]{2}{*}{ Jung, M } & 473 & Lim, YW & 87 & & \\
\hline & & Lin, $\mathrm{H}-\mathrm{C}$ & 311 & Nachtigall, J & 369 \\
\hline Kajimoto, T & 413 & Lin, L & 79 & Nagai, A & 535,579 \\
\hline Kalthoff, $\mathrm{H}$ & 369 & Liu, $\mathrm{H}$ & 449 & Najahi, E & 499 \\
\hline Kamarudin, MS & 289 & Liu, J & 49,517 & Nakagawa, K & 599 \\
\hline Kaplan, JB & 73 & Liu, K-S & 109 & Nakagawa, Y & 249 \\
\hline Katagiri, T & 565 & Liu, L & 115 & Nakano, C & 551 \\
\hline Kawaguchi, M & 419 & Liu, $\mathrm{P}$ & 523 & Nakazawa, T & 377 \\
\hline Kawahara, T & $45,147,373,535,579$ & Liu, S & 271 & Nallet, J-P & 499 \\
\hline Kawamoto, K & 419 & Liu, $\mathrm{X}$ & 79,433 & Nam, S-J & 593 \\
\hline Kawasaki, H & 263 & Liu, X-Z & 143 & Namatame, M & $113,169,197$ \\
\hline Kawatani, M & 123 & Liu, Y & $175,513,539$ & Narasaki, R & 91,361 \\
\hline Kawecki, R & 219 & Loesgen, S & 593 & Nepveu, F & 499 \\
\hline Kazufumi, T & 277 & LoVetri, K & 73 & $\mathrm{Ng}, \mathrm{S}$ & 275 \\
\hline
\end{tabular}




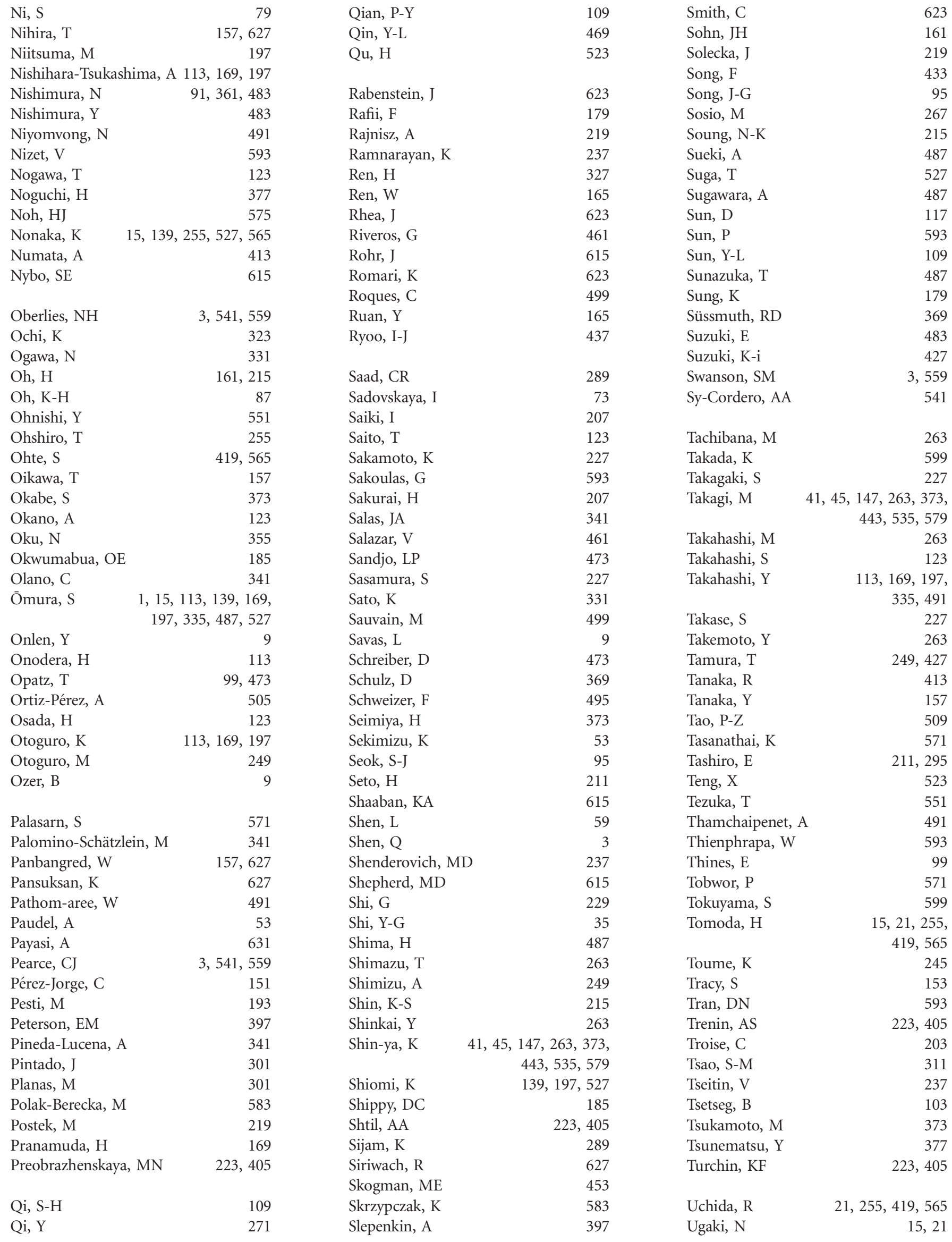


Uramoto, M

Ur-Rehman, T

van Niekerk, AC

Virág, E

Vouloumanou, EK

Vuorela, PM

Vurro, M

Wang, B

Wang, $\mathrm{H}$

Wang, H-L

Wang, H-Q

Wang, J

Wang, M-G

Wang, N

Wang, Q

Wang, R

Wang, S

Wang, $\mathrm{Y}$

Wang, Y-F

Wang, Z

Wani, MC

Waśko, A

Watanabe, $\mathrm{H}$

Watanabe, $\mathrm{K}$

Watanabe, Y

Watanapokasin, $\mathrm{R}$

Weber, A

Wei, D-Q

Weinstein, RN
123

397

609

193

175

453

203

479

$67,79,229$

143

509

433

35

317

449

175

229, 317

79, 523

109

523

3, 559

583

355

377

169

295

153

469

381
Wen, C-N

Wright, GD

Wu, D-J

$\mathrm{Wu}, \mathrm{L}$

$\mathrm{Wu}, \mathrm{S}$

Wu, X-J

Wu, Y

Xiao, C

Xie, Y-Y

$\mathrm{Xu}, \mathrm{A}$

$\mathrm{Xu}, \mathrm{H}-\mathrm{Z}$

$\mathrm{Xu}, \mathrm{L}-\mathrm{H}$

$\mathrm{Xu}, \mathrm{Q}$

Xue, X

Yamada, H

Yamada, T

Yamamoto, K

Yamamoto, $\mathrm{T}$

Yamamura, $\mathrm{H}$

Yamazaki, $\mathrm{H}$

Yang, H-F

Yang, J

Yang, $\mathrm{K}$

Yang, $\mathrm{X}$

Yang, $\mathrm{Y}$

Yao, T-E

Ye, Y

Yeom, J-H

Yoganathan, K
Yokoshima, S

53

591

311

79

117

35

67

513, 539

327

117

327

469

49

129

113, 197

227, 413

211, 295

527

$249,373,427$

$15,21,419,565$

531

559

449

115

513, 539

327

307, 531

95

275
Yoo, I-D

437

Yoo, JK

Yoshida, M

437

Yu, J-C

$\mathrm{Yu}, \mathrm{L}$

Yun, B-S

Zajko, J

Zetterström, CE

Zhanel, GG

Zhang, F

Zhang, G

Zhang, H-W

Zhang, J

Zhang, L

Zhang, N

Zhang, S

Zhang, W

Zhang, $\mathrm{X}$

Zhang, X-Y

Zhao, G-Z

Zhao, J

Zheng, $\mathrm{X}$

Zheng, Z

Zhou, Y

Zhu, W

Zhu, Y-l

Zokaeifar, $\mathrm{H}$

Zonno, MC

Zou, Z-M
263, 591

35

513,539

87,95

219

397

495

479

517

143

35

115,433

117

469

479

229,449

35, 109

469

479

513,539

49

129, 165

523

307

289

203

143 